\title{
Safety and efficacy of salvage low-dose-rate brachytherapy for prostate bed recurrences following radical prostatectomy
}

\author{
Aryavarta M.S. Kumar, MD, PhD', Kristin L. Smith, BS', Chandana A. Reddy, MS', Kevin L. Stephans, MD!, \\ Eric A. Klein, MD², Jay P. Ciezki, MDl \\ 'Department of Radiation Oncology, ${ }^{2}$ Glickman Urologic and Kidney Institute, Cleveland Clinic, Cleveland, USA
}

\begin{abstract}
Purpose: To report efficacy in our series of nodular recurrences in the post-surgical bed that underwent salvage low-dose-rate (LDR) brachytherapy.

Material and methods: Patients with radical prostatectomy (RP) who had biochemical failure with nodular recurrence detected by DRE, ultrasound, and pelvic CT and then salvaged with LDR ${ }^{125} \mathrm{I}$ brachytherapy were included. Nodular recurrences were biopsy confirmed adenocarcinoma, and patients had no evidence of nodal or distant metastasis on imaging including bone scan. Follow up was at least every 6 months with a serial prostate specific antigen (PSA).

Results: Twelve patients had salvage LDR brachytherapy with median age 69 years (range 59-86) and median presalvage PSA of $4.22 \mathrm{ng} / \mathrm{ml}$. Nodule biopsy Gleason score was 7, 8, or undifferentiated. Median rectal $\mathrm{V}_{100}$ was 0.00 cc. Compared to pre-salvage, patients reported no additional genitourinary (GU) toxicity. After a median 35 months postsalvage follow up (range 10-81 months), patients had a median PSA nadir of $0.72 \mathrm{ng} / \mathrm{ml}$ (range 0.01-22.4). At 6 months post salvage, $90 \%$ of patients had a PSA below pre-salvage levels. At last follow up, 4 patients had PSA control.

Conclusions: There was a trend to improved biochemical relapse free survival for lower Gleason score and pre-salvage PSA, which may be indicative of the lack of or only low volume metastatic disease. LDR brachytherapy is an effective salvage technique and can be considered in well selected patients allowing for dose escalation to the nodular recurrence.

Key words: LDR brachytherapy, nodular recurrence, post-prostatectomy, prostate cancer, salvage brachytherapy.

\section{Purpose}

The risk of recurrence after radical prostatectomy for non-metastatic prostate cancer is approximately $10 \%$ at 10 years and up to $40 \%$ for patients with positive margins and extraprostatic extension [1,2]. Among men receiving prostatectomy, the biochemical recurrence rate (greater than $0.4 \mathrm{ng} / \mathrm{ml}$ ) was $34 \%, 37 \%$ and $37 \%$, and prostate cancer specific mortality (PCSM) was 3\%, $11 \%$, and $21 \%$ at 5,10 , and 15 years, respectively [3]. Some of these recurrences are true local failures that manifest as an elevated prostate specific antigen (PSA) and/or a biopsy proven nodule. For those recurrences that occur in the prostatectomy bed without evidence of metastatic spread, external beam radiation therapy (EBRT) with or without androgen deprivation has traditionally been recommended [4]. However, despite highly conformal EBRT techniques that can salvage many patients, adjacent organs have a higher risk of acute and long-term toxicity from EBRT; this includes side effects involving the bladder and rectum, and possible radiation-induced second malignancies [5].

While low-dose-rate (LDR) prostate brachytherapy has traditionally been used only as primary therapy for prostate cancer, we have previously demonstrated the technical feasibility of LDR brachytherapy as an alternative strategy for the management of local recurrences that are visible with medical imaging (i.e. computed tomography (CT), magnetic resonance imaging (MRI), ultrasound) after radical prostatectomy (RP) [6]. Compared to salvage EBRT, LDR brachytherapy has the advantage of significantly lower radiation dose to surrounding structures. In this report we update our experience on the safety of salvage LDR brachytherapy and now report on its efficacy. 


\section{Material and methods}

\section{Patient selection}

Twelve consecutive patients with biopsy-proven prostatic fossa recurrences visible on medical imaging after radical prostatectomy underwent salvage LDR brachytherapy with ${ }^{125}$ I from December 2006 to December 2011. The initial surgical approaches were radical retropubic prostatectomy, radical perineal prostatectomy, or robotic prostatectomy. All recurrences were documented by digital rectal examination, ultrasonography, biopsy, and abdominal/pelvic CT. Patients were selected for salvage LDR brachytherapy because of a favorable nodule location anterior to the rectum and not involving the bladder that would allow favorable dosimetry. Metastatic workup was negative by bone scan and/or CT. After surgery and prior to salvage LDR brachytherapy, two patients had re- ceived EBRT, 1 patient had androgen deprivation therapy (ADT), and one patient had high intensity focused ultrasound (HIFU). After treatment, serum PSA was obtained every 6 months. Biochemical failure was defined by nadir $+2 \mathrm{ng} / \mathrm{ml}$. Toxicity was assessed every 6 months using a template based physician assessment. Genitourinary (GU) and gastrointestinal (GI) toxicity was also reviewed from medical oncology and primary care visits.

\section{Dosimetry and implant technique}

Briefly, our implant technique was adapted from the American Brachytherapy Society (ABS) [7] with slight modifications to the technique used for intact prostate to accommodate the perirectal location of the local recurrences, as was described previously [6]. Patients were positioned in exaggerated dorsal lithotomy, the rectum

Table 1. Summary of patient, disease characteristics, pre-LDR brachytherapy and post-LDR brachytherapy prostate specific antigen, and disease status

\begin{tabular}{|c|c|c|c|}
\hline Characteristics & Median & Minimum & Maximum \\
\hline Age (yrs) & 69 & 59 & 86 \\
\hline Pre-LDRB PSA (ng/ml) & 4.22 & 0.55 & 15.2 \\
\hline Time from RP to LDRB (mth) & 96.5 & 6 & 215 \\
\hline \# PSAs post-LDRB & 8.5 & 1 & 12 \\
\hline PSA follow-up time (mth) & 35 & 10 & 81 \\
\hline PSA nadir (ng/ml) & 0.72 & 0.01 & 22.4 \\
\hline PSA velocity (ng/ml/mth) & 0.374 & -0.051 & 16.477 \\
\hline \multirow[t]{2}{*}{ PSADT (mth) } & 2.6 & -15.7 & 23.1 \\
\hline & & \multicolumn{2}{|c|}{$n(\%)$} \\
\hline \multicolumn{4}{|l|}{ Additional therapy after RP } \\
\hline Androgen deprivation therapy (ADT) & & \multicolumn{2}{|c|}{$1(8.3)$} \\
\hline External beam radiotherapy (EBRT) & & \multicolumn{2}{|c|}{$2(16.7)$} \\
\hline High intensity focused ultrasound (HIFU) & & \multicolumn{2}{|c|}{$1(8.3)$} \\
\hline None & & \multicolumn{2}{|c|}{$8(66.7)$} \\
\hline \multicolumn{4}{|l|}{ Gleason score for nodule } \\
\hline 7 & & \multicolumn{2}{|c|}{$4(33.3)$} \\
\hline 8 & & \multicolumn{2}{|c|}{$5(41.7)$} \\
\hline High grade & & \multicolumn{2}{|c|}{$2(16.7)$} \\
\hline Poorly differentiated & & \multicolumn{2}{|c|}{$1(8.3)$} \\
\hline \multicolumn{4}{|l|}{ Biochemical failure $(n+2)$} \\
\hline No & & \multicolumn{2}{|c|}{$3(25.0)$} \\
\hline Yes & & \multicolumn{2}{|c|}{$8(66.7)$} \\
\hline Not enough data & & \multicolumn{2}{|c|}{$1(8.3)$} \\
\hline \multicolumn{4}{|l|}{ Distant metastases } \\
\hline No & & \multicolumn{2}{|c|}{$6(50)$} \\
\hline Yes & & \multicolumn{2}{|c|}{$6(50)$} \\
\hline \multicolumn{4}{|l|}{ Status } \\
\hline Alive with no evidence of disease (ANED) & & \multicolumn{2}{|c|}{$4(33.3)$} \\
\hline Alive with biochemical failure (AbF) & & \multicolumn{2}{|c|}{$2(16.7)$} \\
\hline Alive with disease (AWD) & & \multicolumn{2}{|c|}{$6(50)$} \\
\hline
\end{tabular}

PSA - prostate specific antigen, LDRB - low-dose-rate brachytherapy, yrs - years, mth-months, PSADT - PSA doubling time, RP - radical prostatectomy, ADT-androgen deprivation therapy, EBRT - external beam radiotherapy, HIFU - high intensity focused ultrasound, ANED - alive with no evidence of disease, AbF - alive with biochemical failure, AWD - alive with disease 
A

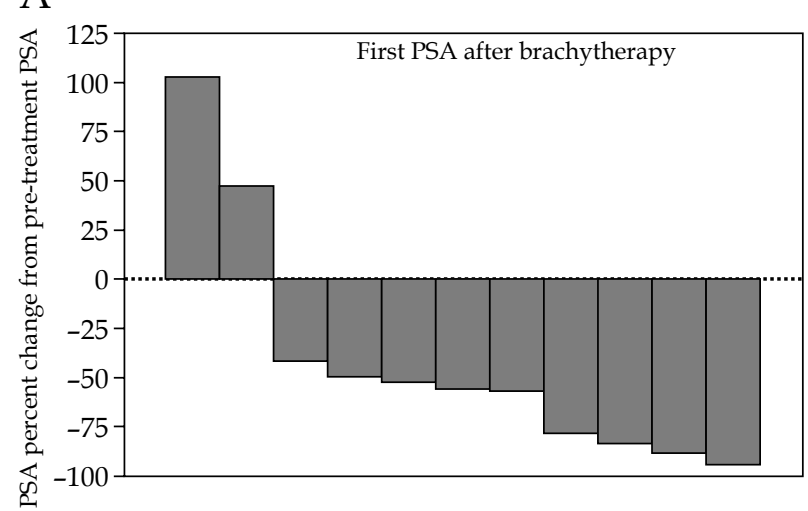

C

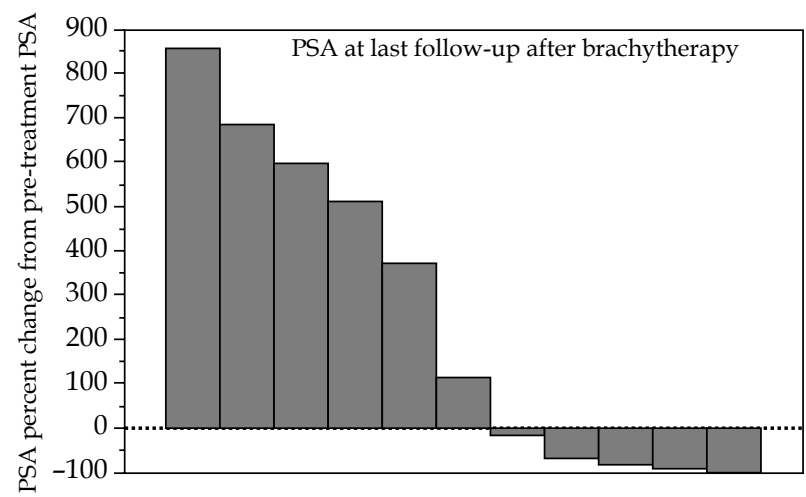

was irrigated, and a transrectal ultrasound probe visualized the nodule was inserted. The brachytherapy plan was generated using VariSeed (v7.1, Varian Medical Systems, Palo Alto, CA, USA) with a prescribed dose of 144 Gy. The prescription target was the nodule with a small $3-5 \mathrm{~mm}$ margin. Seeds were implanted into the nodule. Except for perirectal sources, all sources were stranded or linked
B

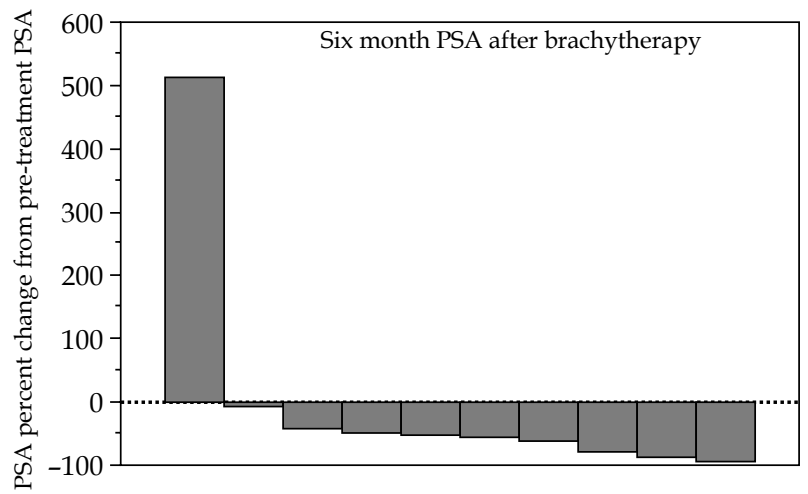

Fig. 1. A) Waterfall plot of individual percent pre-treatment PSA change at first follow up. B) Waterfall plot of individual percent pre-treatment PSA change at 6 months. C) Waterfall plot of individual percent pre-treatment PSA change at last follow-up

(Oncura RAPID Strand [Oncura Inc., Arlington Heights, IL, USA] or CR Bard RediLink (Bard Inc., Medical Division, Covington, GA, USA), respectively. Activity ranged between 0.38-0.48 U/source. The rectal volume receiving $100 \%\left(\mathrm{rV}_{100}\right)$ of the prescribed dose was kept $<1 \mathrm{~cm} \mathrm{[4].}$ Source needle insertion was performed in a manner similar to a typical prostate implantation.

Table 2. Tumor factors, PSA kinetics, and status at last follow up for each patient

\begin{tabular}{ccccccccc} 
Patient & Gleason & $\begin{array}{c}\text { Pre-LDRB } \\
\text { PSADT }(\mathrm{mth})\end{array}$ & $\begin{array}{c}\text { PSA prior to } \\
\text { LDRB }\end{array}$ & $\begin{array}{c}\text { Time from RP } \\
\text { to LDRB }(\mathrm{mth})\end{array}$ & $\begin{array}{c}\text { Post-LDRB } \\
\text { PSADT (mth) }\end{array}$ & $\begin{array}{c}\text { Post-LDRB } \\
\text { f/u (mth) }\end{array}$ & $\begin{array}{c}\text { Status at last } \\
\text { follow up }\end{array}$ \\
\hline 1 & 7 & 14.1 & 6.04 & 88 & 8.6 & 63 & AWD \\
\hline 2 & 8 & 8.1 & 9.47 & 70 & $6.4^{*}$ & 14 & AWD \\
\hline 3 & 7 & 16.7 & 6.5 & 151 & -15.7 & 63 & ANED \\
\hline 4 & 8 & 5.7 & 15.2 & 215 & $2.1^{*}$ & 10 & AWD \\
\hline 5 & 7 & 23.1 & 4.53 & 182 & 23.1 & 81 & AbF \\
\hline 6 & 8 & 5.7 & 2.27 & 40 & $2.4^{*}$ & 36 & AbF \\
\hline 7 & Poorly diff.\# & 3.2 & 2.19 & 31 & $2.8^{*}$ & 47 & AWD \\
\hline 8 & 8 & 9.4 & 3.91 & 165 & -11.6 & 34 & ANED \\
\hline 9 & 8 & 70.1 & 0.55 & 105 & $13.7^{*}$ & 33 & AWD \\
\hline 10 & Poorly diff.\# & NA & 0.72 & 174 & NA & 27 & AWD \\
\hline 11 & 7 & 3.2 & 4.56 & 6 & -12.9 & 53 & ANED \\
\hline 12 & Poorly diff.\# & 8 & 2.36 & 69 & NA & 12 & ANED \\
\hline
\end{tabular}

\#Gleason score not assigned and reported as poorly differentiated/high grade adenocarcinoma. *Patients received ADT. PSADT was calculated before ADT was initiated. Other abbreviations - see Table 1. 


\section{Statistical analysis}

Descriptive and comparative statistics were done using SAS v9.2 (SAS Institute, Cary, NC, USA). Cox Proportional Hazard Regression analysis was used to identify factors associated with biochemical failure. Disease free survival rates were estimated from Kaplan-Meier analyses. Statistical significance was defined as $p<0.05$.

\section{Results}

Patient and disease characteristics for the 12 patients prior to salvage LDR brachytherapy are reported in Table 1. Four patients (33.3\%) had Gleason score of 7 and 5 patients $(41.7 \%)$ had a Gleason Score of 8 . Three patients (25\%) had no assigned Gleason score due to treatment effect from previous ADT or EBRT and were described as either high grade or poorly differentiated. Median $\mathrm{V}_{100}$ for rectum was 0.00 cc (range: $0.00-0.1 \mathrm{cc}$ ).

Tabulated PSA follow-up appears in Table 1 and 2. One patient was on androgen deprivation before and after LDR brachytherapy, and was omitted from PSA follow-up analysis. Thus, out of 11 patients, 9 patients $(81.8 \%)$ had an immediate PSA decrease after salvage treatment (Figure 1A). At 6 months post-LDR brachytherapy, 10 patients had 6 month or greater follow-up, and nine (90\%) continued to have a post-salvage PSA that was lower than the pre-salvage PSA (Figure 1B). The one patient who had an increase at 6 months had a high pre-salvage PSA of 15.2 that continued to increase after salvage LDR brachytherapy. Median biochemical relapse free survival (bRFS) post-salvage was 23 months. At last follow-up, four patients had a lower PSA compared to pre-LDR brachytherapy (Figure $1 \mathrm{C}$ and Table 1). When stratifying by Gleason score (Figure 2), there was a trend for bRFS benefit for lower Gleason score. Median distant metastases-free survival was 55 months. Two patients subsequently had distant metastases in the pelvic lymph nodes at 17 and 55 months after salvage brachytherapy (Figure 3). At last follow-up,

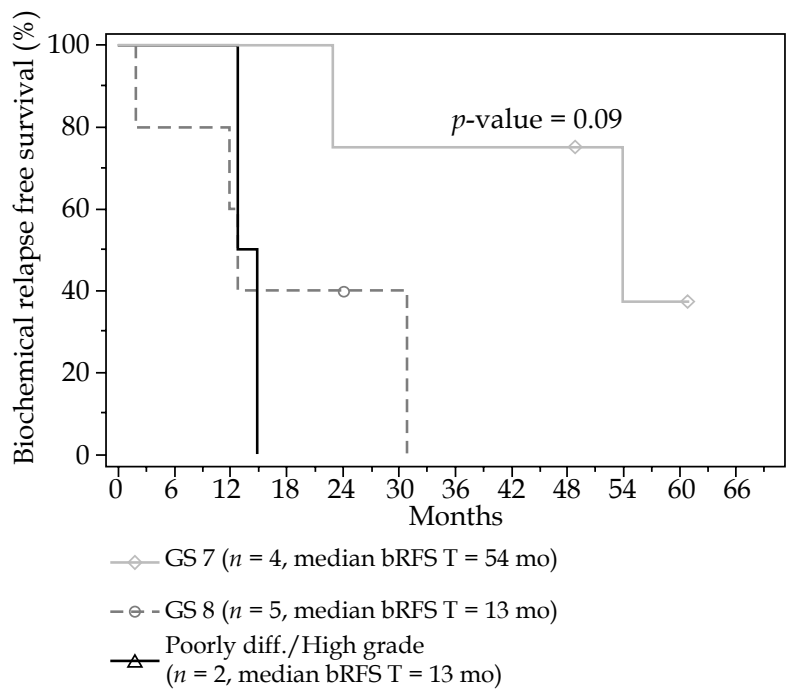

Fig. 2. Biochemical relapse free survival (bRFS) after salvage brachytherapy by Nodule Gleason Score $(n=11)$ four patients ( $33 \%$ ) were alive with no evidence of disease (ANED) with initial PSA (ng/ml) 6.5, 4.56, 3.91, and 2.36; these PSA values were not significantly different than the patients who had biochemical and/or distant failures.

We also considered PSA doubling time (PSADT) before and after salvage LDR brachytherapy to determine if there may be a change in disease progression in those patients that had biochemical failure. Table 1 lists the PSADT and follow-up in months for each patient. In 9 (75\%) patients, PSADT post-LDR brachytherapy decreased compared to PSADT pre-LDR brachytherapy. In general, PSADT decreased for all patients who have had disease progression (biochemical failure, distant metastatic disease).

There was no statistically significant correlation between patients that had biochemical failure and patient age, prostatectomy Gleason score, nodule Gleason score, PSA at time of salvage, or time from initial diagnosis to recurrence.

Eleven out of 12 patients in this series have not reported a Grade 2 or higher gastrointestinal or genitourinary toxicity after salvage LDR brachytherapy. One patient who had salvage ADT, LDR brachytherapy, and additional ADT had a cystoscopy for evaluation of mild hematuria and urinary incontinence; some of this incontinence was present prior to salvage LDR brachytherapy, however, his symptoms worsened. He ultimately received a prosthetic urinary sphincter for his symptoms.

\section{Discussion}

External beam radiotherapy has an established role in the management of prostate cancer after radical prostatectomy. There is level 1 evidence demonstrating that it improves biochemical relapse free survival in post prostatectomy patients with adverse pathological features [8] and also substantial evidence of a beneficial effect from salvage radiotherapy in those with a low but detectable PSA [9]. The advantages to EBRT in the adjuvant and salvage settings are a significantly increased biochemical

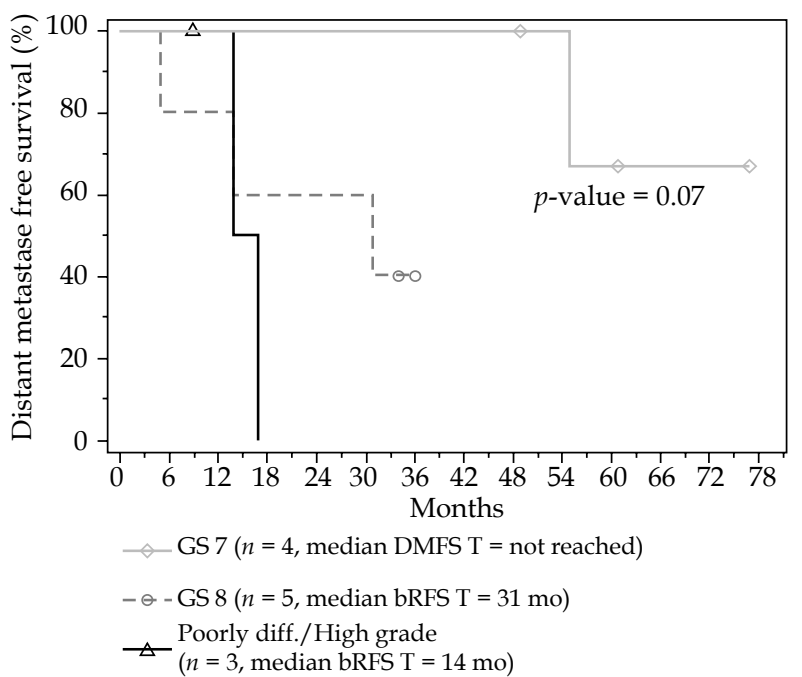

Fig. 3. Distant metastases free survival (DMFS) after salvage brachytherapy by Nodule Gleason Score $(n=12)$ 
control rate that may help reduce distant metastases and improve survival, and the disadvantages are increased GI and GU toxicity. While the optimal post-prostatectomy EBRT dose has not been established, ASTRO/AUA (American Society for Radiation Oncology/American Urological Association) guidelines recommend a minimum total radiation dose of 64-65 Gy with clinical data suggesting that higher total doses may lead to improved tumor outcome [10]. In the setting of a nodular recurrence however, there is likely a need for increased dose as there is gross disease present and there is a correlation of total dose to tumor burden [11]. Adjacent structures to the nodule however, will limit the total radiation dose that can be safely delivered with EBRT. This is an ideal scenario for brachytherapy. It remains an open question whether there is a role for combined salvage EBRT with brachytherapy, a strategy that would maximize the advantages of EBRT in covering a wide field and of the higher dose deliverable by LDR brachytherapy; the downside of this approach is the added toxicity of EBRT.

In this experience, we tested the hypothesis that dose escalation using salvage LDR brachytherapy alone in the setting of a nodular recurrence would result in meaningful benefits with minimal toxicity. The use of brachytherapy has been described in the literature following recurrences after definitive EBRT [12-14], however, there are only a few reports of salvage brachytherapy after prostatectomy [15-18]. Other salvage options include cryotherapy [19] and HIFU [20,21] but recent experiences describe a limited success with a $5-10 \%$ incidence of severe cases of urinary toxicity requiring surgical intervention and biochemical control rates of $\sim 50 \%$. A combination of EBRT with HDR intensity modulated brachytherapy (IMBT) has also been reported with biochemical non-evidence of disease (bNED) of $30-40 \%$ [15]. Our experience demonstrates that with careful dosimetry planning, salvage LDR brachytherapy for post-prostatectomy nodular recurrences can be delivered with minimal acute and late toxicity. Additionally, in those patients with previous local salvage (HIFU/EBRT), we noted no significant increased toxicity compared to patients with no previous local salvage treatment.

Assessing the efficacy of therapy for local recurrences after radical prostatectomy is challenging due to the insensitivity of imaging techniques needed to reliably exclude the presence of metastatic disease. Of our 12 patients, 9 had a decreased PSADT after salvage LDR brachytherapy, including 1 patient with PSADT that went from $>70$ months to 13.7 months. These decreases represent a shorter time to PSA doubling and thus accelerating disease progression. Two patients who remain disease and biochemical failure free have a negative PSADT post-LDR brachytherapy representing PSA control. While treating a local recurrence can reduce the disease burden in a patient, we would expect an increase in PSADT if the LDR brachytherapy significantly influenced the progression of PSA failure.

At the time of analysis, four patients (33\%) were alive without evidence of biochemical or distant failure at 1263 months after salvage LDR brachytherapy, suggesting that they had local disease only. Of these 4 patients, 2 had
Gleason 7 disease, 1 had Gleason 8 , and 1 had high grade with no Gleason reported; all 4 of these patients had PSA levels at the time of salvage $\leq 6.5$. The best candidates for salvage LDR brachytherapy appear to be those with lower Gleason grades and PSA at time of salvage, which is likely indicative of the lack of or only low volume metastatic disease. Further subgroup analysis was limited by the small numbers of patients in this report.

Two other groups have described their experience with salvage brachytherapy for nodular recurrences after prostatectomy. Losa et al. published on their series of 10 patients who had prostatectomy; this was followed by external beam in $8(80 \%)$ patients and all 10 had subsequent brachytherapy. The pre-brachytherapy PSA range was 1.1-6.1 ng/ml. With median follow-up of 20.6 months, the reported 5-year biochemical disease free survival (bDFS) was $88.6 \%$ [16]. This study used external beam radiotherapy prior to brachytherapy and at least one of the patients on study $(10 \%)$ developed toxicity from EBRT. In a Spanish study of 42 patients with median follow-up of 26 months, salvage brachytherapy was noted to be safe and effective with five year bDFS of $88.6 \%$ [18]. The initial biochemical failure was defined as $\geq 0.4 \mathrm{ng} / \mathrm{ml}$ with a biopsy proven recurrence while biochemical failure after salvage brachytherapy was defined as PSA nadir $+2 \mathrm{ng} / \mathrm{ml}$. In both experiences, there were no reported grade 3 or higher toxicity from salvage LDR brachytherapy and there were no predictive factors that were reported to indicate patients who most benefit from salvage LDR brachytherapy. We report that higher Gleason score is associated with a higher risk of biochemical failure, however, in the Losa and Gomez-Veiga studies, the Gleason is predominantly Gleason 6 or 7 disease or not reported; this may explain the differences in reported biochemical failure rates.

Detection of nodular recurrences can be challenging; however, emerging data indicates that pelvic dynamic contrast enhanced (DCE) MRI can aid in the detection of residual prostate tissue and/or nodular recurrences [22]. With increased imaging resolution, there may be a higher incidence of this clinical presentation. We believe that in well selected patients with nodular recurrences and low pre-salvage PSA, salvage LDR brachytherapy is an excellent treatment strategy to dose escalate for local control and while not statistically significant would recommend caution in this approach for patients with Gleason $>7$. Most importantly however, further efforts are also needed to help identify other non-local sites of failure to better select candidates for LDR brachytherapy.

While this report represents a unique salvage option for patients with nodular recurrence only, limitations of the study include its small size and lower statistical power to identify potential subgroups that may have the most benefit. Additionally, the common biases that are present in retrospective reports are also present here. These patients represent a small subset of all prostate cancer patients that recur, however, because of the scarcity of published reports, small series are valuable to guide recommendations for the clinician. Additionally with limited published evidence and follow-up, we believe this small series improves our understanding of LDR bra- 
chytherapy. Additional follow-up and study are needed to validate the findings from this report.

\section{Conclusions}

Salvage brachytherapy is technically feasible and presents a treatment option to dose escalate a nodular recurrence in the prostate bed. There is minimal gastrointestinal and genitourinary toxicity with the additional advantages of lower cost and patient convenience. Well selected patients can be considered for salvage LDR brachytherapy.

\section{Disclosure}

Authors declare no conflict of interest.

\section{References}

1. Catalona WJ, Smith DS. 5-year tumor recurrence rates after anatomical radical retropubic prostatectomy for prostate cancer. J Urol 1994; 152: 1837-1842.

2. Lu J, Wirth GJ, Wu S et al. A close surgical margin after radical prostatectomy is an independent predictor of recurrence. J Urol 2012; 188: 91-97.

3. Uchio Em, Aslan M, Wells CK et al. Impact of biochemical recurrence in prostate cancer among US veterans. Arch Intern Med 2010; 170: 1390-1393.

4. Macdonald OK, Schild SE, Vora SA et al. Salvage radiotherapy for palpable, locally recurrent prostate cancer after radical prostatectomy. Int J Radiat Oncol Biol Phys 2004; 58: 1530-1535.

5. Phillips R. Prostate cancer: post-treatment complications of radiotherapy and prostectomy. Nat Rev Urol 2014; 11: 129.

6. Traudt K, Ciezki J, Klein EA. Low-dose-rate brachytherapy as salvage treatment of local prostate cancer recurrence after radical prostatectomy. Urology 2011; 77: 1416-1419.

7. Davis BJ, Horwitz EM, Lee WR et al. American Brachytherapy Society consensus guidelines for transrectal ultrasound-guided permanent prostate brachytherapy. Brachytherapy 2012; 11: 6-19.

8. Patel P, Lee WR. Radiotherapy following radical prostatectomy. Expert Rev Anticancer Ther 2012; 12: 973-979.

9. Stephenson AJ, Scardino PT, Kattan MW et al. Predicting the outcome of salvage radiation therapy for recurrent prostate cancer after radical prostatectomy. J Clin Oncol 2007; 25: 20352041.

10. Thompson IM, Valicenti RK, Albertsen P et al. Adjuvant and Salvage Radiotherapy After Prostatectomy: AUA/ASTRO Guideline. J Urol 2013; 190: 441-449.

11. King CR, Spiotto MT. Improved outcomes with higher doses for salvage radiotherapy after prostatectomy. Int J Radiat Oncol Biol Phys 2008; 71: 23-27.

12. Aaronson DS, Yamasaki I, Gottschalk A et al. Salvage permanent perineal radioactive-seed implantation for treating recurrence of localized prostate adenocarcinoma after external beam radiotherapy. BJU Int 2009; 104: 600-604.

13. Lee HK, Adams MT, Motta J. Salvage prostate brachytherapy for localized prostate cancer failure after external beam radiation therapy. Brachytherapy 2008; 7: 17-21.

14. Chen $C P$, Weinberg V, Shinohara $K$ et al. Salvage HDR brachytherapy for recurrent prostate cancer after previous definitive radiation therapy: 5 -year outcomes. Int $J$ Radiat Oncol Biol Phys 2013; 86: 324-329.

15. Niehoff $P$, Loch T, Nurnberg N et al. Feasibility and preliminary outcome of salvage combined HDR brachytherapy and external beam radiotherapy (EBRT) for local recurrences after radical prostatectomy. Brachytherapy 2005; 4: 141-145.
16. Losa A, Nava LD, Di Muzio N et al. Salvage brachytherapy for local recurrence after radical prostatectomy and subsequent external beam radiotherapy. Urology 2003; 62: 10681072.

17. Gaztanaga M, Crook JM. Permanent seed brachytherapy for locally recurrent prostate cancer after radical prostatectomy: a case report and review of the literature. Brachytherapy 2012; 12: $338-342$

18. Gomez-Veiga F, Marino A, Alvarez L et al. Brachytherapy for the treatment of recurrent prostate cancer after radiotherapy or radical prostatectomy. BJU Int 2012; 109 Suppl 1: 17-21.

19. Siddiqui SA, Mynderse LA, Zincke H et al. Treatment of prostate cancer local recurrence after radical retropubic prostatectomy with 17-gauge interstitial transperineal cryoablation: initial experience. Urology 2007; 70: 80-85.

20. Asimakopoulos AD, Miano R, Virgili G et al. HIFU as salvage first-line treatment for palpable, TRUS-evidenced, biopsy-proven locally recurrent prostate cancer after radical prostatectomy: a pilot study. Urol Oncol 2012; 30: 577-583.

21. Murota-Kawano A, Nakano M, Hongo S et al. Salvage highintensity focused ultrasound for biopsy-confirmed local recurrence of prostate cancer after radical prostatectomy. BJU Int 2010; 105: 1642-1645.

22. Rischke HC, Schafer AO, Nestle U et al. Detection of local recurrent prostate cancer after radical prostatectomy in terms of salvage radiotherapy using dynamic contrast enhancedMRI without endorectal coil. Radiat Oncol 2012; 7: 185-192. 